Jan Malý*, Department of Mathematical Analysis, Charles University,

Sokolovská 83, 18600 Praha,, Czech Republic

e-mail: maly@@karlin.mff.cuni.cz

\title{
THE DARBOUX PROPERTY FOR GRADIENTS
}

\begin{abstract}
It is well known that the derivative of a function of one variable has the Darboux property. In this paper it is shown that the gradient of a differentiable function of several variables maps certain closed convex sets to connected sets.
\end{abstract}

It is well known that any differentiable real function $f$ on an interval $I \subset \mathbb{R}$ has the Darboux property. This means that if $a<b$ are points in $I$ and $\xi$ is a value between $f^{\prime}(a)$ and $f^{\prime}(b)$, then there is $x \in[a, b]$ such that $f^{\prime}(x)=\xi$. It is equivalent to say that for any closed convex subset $K$ of $I$ the image $f^{\prime}(K)$ is connected. In this note we are going to show that an analogous property holds for the derivative (gradient) of a differentiable function of several variables. Even more generally, we work in infinite-dimensional Banach spaces.

As a special case of the result we obtain the Darboux property of partial derivatives of differentiable functions, which is due to Neugebauer $[\mathrm{N}]$ and Weil [W]. Let us mention that if we modify the definition of Darboux property of partial derivatives as in $[\mathrm{N}]$, the assumption of differentiability may be weakened.

The one-dimensional Darboux property has been generalized in a variety of other directions as well. There are several papers which are devoted to the Darboux property of derivatives of interval functions of several variables. A general result was proved by Mišik $[\mathrm{M}]$, for further development see $[\mathrm{N}],[\mathrm{B}]$.

We suppose that $X$ is a Banach space. In particular we may consider $X=\mathbb{R}^{n}$. The symbol $U(x, r)$ is used for the open ball with center at $x$ and

Key Words: Darboux property, differentiable, gradient

Mathematical Reviews subject classification: Primary: 26B05

Received by the editors November 13, 1995

*I thank Clifford E. Weil, Luděk Zajíček and Petr Holický for valuable discussions. 
radius $r$. We denote the dual space of $X$ by $X^{*}$. Both norms in $X$ and $X^{*}$ are denoted by $\|\ldots\|$. We write $x^{*} \cdot x$ for the duality pairing between $x^{*} \in X^{*}$ and $x \in X$. The topological notions in $X$ and in $X *$ are with \& respect to the corresponding norm topology. Differentiability is interpreted as Fréchet differentiability. This means that a function $f$ is said to be differentiable at $x \in X$ with respect to $D \subset X$ if there is a unique $x^{*} \in X^{*}$ (called the derivative of $f$ at $x$ with respect to $D$ ) such that

$$
\lim _{y \rightarrow x, y \in D} \frac{f(y)-f(x)-x^{*} \cdot(y-x)}{\|y-x\|}=0 .
$$

If $f$ is differentiable with respect to its domain, we say simply that $f$ is differentiable and denote the derivative by $f^{\prime}$. The main goal of this note is the following theorem.

Theorem 1 Let $f$ be a differentiable function on $D \subset X$. Then for any closed convex set $K \subset D$ with nonempty interior, $f^{\prime}(K)$ is a connected subspace of $X^{*}$.

For the proof of Theorem 1, we may assume that $D=K$. We fix a closed convex set $K \subset X$ with nonempty interior and start with a series of auxiliary results.

Lemma 2 (Ekeland's variational principle) Let $g$ be a continuous function on $\bar{U}(x, r) \subset X$ and $\varepsilon>0$. Suppose that

$$
g(y) \leq g(x)+\varepsilon r
$$

for each $y \in \bar{U}(x, r)$. Then there is $u \in \bar{U}(x, r / 2)$ such that

$$
g(y) \leq g(u)+2 \varepsilon|y-u|
$$

for each $y \in \bar{U}(x, r)$.

Proof. We refer e.g. to $[\mathrm{Ph}]$, Lemma 3.13, but for reader's convenience we notice that the finite-dimensional case is easy. Indeed, we find $u \in \bar{U}(x, r)$ such that $y \mapsto g(y)-2 \varepsilon|y-x|$ attains a maximum at $u$ relative to $\bar{U}(x, r)$. Then an exercise in handling the triangle inequality shows that in fact $u \in \bar{U}(x, r / 2)$ and (1) holds.

Since $K$ has a nonempty interior, we may fix a ball $\bar{U}\left(x_{0}, r_{0}\right)$ inside $K$. 
Lemma 3 Let $x \in K$ and $r>0$. Then there are $x_{1} \in K$ and $r_{1}>0$ such that

$$
r_{1} \geq \min \left\{\frac{r r_{0}}{2\left\|x-x_{0}\right\|}, \frac{r}{2}, r_{0}\right\} \text { and } \bar{U}\left(x_{1}, r_{1}\right) \subset K \cap \bar{U}(x, r) .
$$

Proof. If $\left\|x-x_{0}\right\| \leq r / 2$, then it is enough to set

$$
x_{1}=x_{0}, \quad r_{1}=\min \left\{r_{0}, r / 2\right\} .
$$

Let $\left\|x-x_{0}\right\| \geq r / 2$. We set

$$
x_{1}=x+\frac{1}{2} r \frac{x_{0}-x}{\left\|x_{0}-x\right\|} \text { and } r_{1}=\min \left\{\frac{r}{2}, \frac{r r_{0}}{2\left\|x-x_{0}\right\|}\right\} .
$$

Now, each point $y_{1}$ from $\bar{U}\left(x_{1}, r_{1}\right)$ is a convex combination of $x$ and a point $y_{0} \in \bar{U}\left(x_{0}, r_{0}\right)$ and hence belongs to $K$. Since $r_{1} \leq r / 2$, obviously $y_{1} \in \bar{U}(x, r)$.

In the next lemma, we recall some standard tricks from differentiation theory. If $f$ is a differentiable function on $K$, we let

$$
\begin{aligned}
& E_{i, m, k}(f)=\left\{x \in K:\left\|x-x_{0}\right\| \leq 2^{i} r_{0},\right. \\
& \left.\left[y \in K,\|y-x\|<2^{-m} \Rightarrow\left|f(y)-f(x)-f^{\prime}(x) \cdot(y-x)\right| \leq 2^{-k}\|y-x\|\right]\right\} .
\end{aligned}
$$

Lemma 4 Let $f$ be a differentiable function on $K$ and $i, m, k \in \mathbb{N}$. Then

(a) $\limsup _{y \rightarrow x, y \in E_{i, m, k}}\left\|f^{\prime}(y)-f^{\prime}(x)\right\| \leq 2^{i-k+4}$ for any nonisolated point $x$ of $\bar{E}_{i, m, k}(f)$,

(b) $\bar{E}_{i, m, k}(f) \subset E_{i, m, k-i-5}(f)$.

Proof. Choose $x \in \bar{E}_{i, m, k}(f)$. We find $r \in\left(0, \min \left\{r_{0}, 2^{-m-1}\right\}\right)$ such that

$$
\left|f(y)-f(x)-f^{\prime}(x) \cdot(y-x)\right|<2^{-k}\|y-x\|
$$

for all $y \in \bar{U}(x, r) \cap K$. Notice that then

$$
\left|f\left(y^{\prime}\right)-f(y)-f^{\prime}(x) \cdot\left(y^{\prime}-y\right)\right|<2^{-k+1} r
$$

for all $y, y^{\prime} \in \bar{U}(x, r) \cap K$. If $z \in E_{i, m, k} \cap U(x, r)$ and $y, y^{\prime} \in \bar{U}(x, r) \cap K$, then $\|y-z\|<2 r<2^{-m},\left\|y^{\prime}-z\right\|<2 r<2^{-m}$ and thus also

$$
\left|f\left(y^{\prime}\right)-f(y)-f^{\prime}(z) \cdot\left(y^{\prime}-y\right)\right|<2^{-k}\left(\left\|y^{\prime}-z\right\|+\|y-z\|\right) \leq 2^{-k+2} r .
$$


By Lemma 3 , there are $x_{1} \in K$ and $r_{1}>0$ such that

$$
r_{1} \geq 2^{-i-1} r \text { and } \bar{U}\left(x_{1}, r_{1}\right) \subset K \cap \bar{U}(x, r) .
$$

Let $z \in E_{i, m, k} \cap U(x, r)$ and $h \in X,\|h\|=r_{1}$. Then $x_{1}, x_{1}+h \in K \cap \bar{U}(x, r)$. Using (2) and (3) we obtain

$$
\begin{aligned}
& \left|\left(f^{\prime}(z)-f^{\prime}(x)\right) \cdot h\right|=\mid\left(f\left(x_{1}+h\right)-f\left(x_{1}\right)-f^{\prime}(x) \cdot h\right) \\
& \quad-\left(f\left(x_{1}+h\right)-f\left(x_{1}\right)-f^{\prime}(z) \cdot h\right) \mid \\
& \quad \leq\left|f\left(x_{1}+h\right)-f\left(x_{1}\right)-f^{\prime}(x) \cdot h\right|+\left|f\left(x_{1}+h\right)-f\left(x_{1}\right)-f^{\prime}(z) \cdot h\right| \\
& \quad \leq 2^{-k+1} r+2^{-k+2} r \leq 2^{i-k+4}\|h\| .
\end{aligned}
$$

This proves (a).

Now, we choose $x \in \bar{E}_{i, m, k}$ and $y \in K$ with $\|y-x\|<2^{-m}$. There is a sequence $x_{j}$ of points from $E_{i, m, k}(f)$ converging to $x$. Then for $j$ large enough, and with the aid of (a),

$$
\begin{aligned}
\mid f(y)- & f(x)-f^{\prime}(x) \cdot(y-x) \mid \\
= & \mid\left(f(y)-f\left(x_{j}\right)-f^{\prime}\left(x_{j}\right) \cdot\left(y-x_{j}\right)\right) \\
& -\left(f(x)-f\left(x_{j}\right)-f^{\prime}\left(x_{j}\right) \cdot\left(x-x_{j}\right)\right) \\
& +\left(f^{\prime}\left(x_{j}\right) \cdot(y-x)-f^{\prime}(x) \cdot(y-x)\right) \mid \\
\leq & \left|f(y)-f\left(x_{j}\right)-f^{\prime}\left(x_{j}\right) \cdot\left(y-x_{j}\right)\right| \\
& +\left|f(x)-f\left(x_{j}\right)-f^{\prime}\left(x_{j}\right) \cdot\left(x-x_{j}\right)\right| \\
& +\left|f^{\prime}\left(x_{j}\right) \cdot(y-x)-f^{\prime}(x) \cdot(y-x)\right| \\
\leq & 2^{-k}\left\|y-x_{j}\right\|+2^{-k}\left\|x_{j}-x\right\|+2^{i-k+4}\|y-x\| .
\end{aligned}
$$

Letting $j \rightarrow \infty$ we obtain

$$
\left|f(y)-f(x)-f^{\prime}(x) \cdot(y-x)\right| \leq 2^{i-k+5}\|y-x\|
$$

which proves (b).

Proof of Theorem 1. Let $G^{+}, G^{-} \subset X^{*}$ be open sets such that $f^{\prime}(K) \subset$ $G^{+} \cup G^{-}$and $G^{+} \cap G^{-} \cap f^{\prime}(K)=\emptyset$. We write

$$
F^{+}=\left\{x \in K: f^{\prime}(x) \in G^{+}\right\} \text {and } F^{-}=\left\{x \in K: f^{\prime}(x) \in G^{-}\right\} .
$$


Suppose that both $F^{+}$and $F^{-}$are nonempty. This will lead to a contradiction. Denote $H=\bar{F}^{+} \cap \bar{F}^{-}$. Since $K$ is connected, we deduce that $H \neq \emptyset$. Denote

$$
\begin{aligned}
& F_{i, m, k}^{+}=\left\{y \in E_{i, m, k}(f): \operatorname{dist}\left(f^{\prime}(y), X^{*} \backslash G^{+}\right) \geq 2^{-k+2 i+13}\right\} \\
& F_{i, m, k}^{-}=\left\{y \in E_{i, m, k}(f): \operatorname{dist}\left(f^{\prime}(y), X^{*} \backslash G^{-}\right) \geq 2^{-k+2 i+13}\right\} .
\end{aligned}
$$

Then

$$
K=\bigcup_{i, m, k} F_{i, m, k}^{+} \cup \bigcup_{i, m, k} F_{i, m, k}^{-} .
$$

Using the Baire category theorem in the space $H$, we find $z \in H, \rho_{0}>0$ and $i, m, k \in \mathbb{N}$ such that

$$
H \cap U\left(z, 2 \rho_{0}\right) \subset \bar{F}_{i, m, k}^{+}
$$

or

$$
H \cap U\left(z, 2 \rho_{0}\right) \subset \bar{F}_{i, m, k}^{-}
$$

Assume e.g. that case (4) holds. Also, we may assume that $\rho_{0} \leq r_{0}$ and $3 \rho_{0}<2^{-m}$. From Lemma $4($ a) it follows that

$$
\operatorname{dist}\left(f^{\prime}(y), X^{*} \backslash G^{+}\right) \geq 2^{-k+2 i+13}-2^{-k+i+4}>0
$$

for each $y \in \bar{F}_{i, m, k}^{+}$. In particular

$$
H \cap U\left(z, 2 \rho_{0}\right) \subset F^{+}
$$

Since $H \subset \bar{F}^{-}$, there is a point $x \in F^{-} \cap U\left(z, \rho_{0}\right)$. By $(7), x \notin H$. Let $V$ be the largest ball centered at $x$ such that $V \cap H=\emptyset$. The radius $r$ of $V$ is less than $\rho_{0}$ as $z \notin V$. Hence $\bar{V} \subset U\left(z, 2 \rho_{0}\right)$. Since $K \cap V$ is connected and $V \cap H=\emptyset$, we deduce that $K \cap V \subset F^{-}$. Maximality of $V$ yields that there is a point $w \in U(x, 2 r) \cap U\left(z, 2 \rho_{0}\right) \cap H$. Then, by (4) and (6), $w \in \bar{F}_{i, m, k}^{+}$and

$$
\operatorname{dist}\left(f^{\prime}(w), X^{*} \backslash G^{+}\right) \geq 2^{-k+2 i+12} .
$$

We write

$$
g(y)=f(y)-f(w)-f^{\prime}(w) \cdot(y-w), \quad y \in X
$$

We have

$$
\|y-w\| \leq 3 r \leq 3 \rho_{0}<2^{-m}
$$

for all $y \in \bar{V}$. Since $\left\|x-x_{0}\right\| \leq\left\|z-x_{0}\right\|+\|x-z\| \leq 2^{i+1} r_{0}$, we use Lemma 3 to find a ball

$$
\bar{U}\left(x_{1}, r_{1}\right) \subset K \cap \bar{V}
$$


such that

$$
r_{1} \geq 2^{-i-2} r
$$

By Lemma 4(b),

$$
\bar{F}_{i, m, k}^{+} \subset E_{i, m, k-i-5}(f) .
$$

Thus

$$
|g(y)| \leq 2^{-k+i+5}\|y-w\| \leq 2^{-k+i+7} r \leq 2^{-k+2 i+9} r_{1}
$$

for each $y \in \bar{V}$, which implies that

$$
g(y)-g\left(x_{1}\right) \leq 2^{-k+2 i+10} r_{1}
$$

for each $y \in \bar{U}\left(x_{1}, r_{1}\right)$. By Lemma 2 , there is a point $u \in \bar{U}\left(x_{1}, r_{1} / 2\right)$ such that

$$
g(y) \leq g(u)+2^{-k+2 i+11}\|y-u\|
$$

for all $y \in U\left(x_{1}, r_{1}\right)$, so that

$$
\left\|f^{\prime}(u)-f^{\prime}(w)\right\|=\left\|g^{\prime}(u)\right\| \leq 2^{-k+2 i+11} .
$$

This contradicts (8) because $u \in K \cap V \subset F^{-}$. The proof is complete.

Remark 5 It is not true that $f^{\prime}(L)$ is connected when $L \subset D$ is a line segment. As an example, consider

$$
f\left(x_{1}, x_{2}\right)= \begin{cases}\frac{x_{1} x_{2}^{4}}{x_{1}^{2}+x_{2}^{4}}, & {\left[x_{1}, x_{2}\right] \neq 0} \\ 0, & {\left[x_{1}, x_{2}\right]=0 .}\end{cases}
$$

Then $f$ is differentiable,

$$
\frac{\partial f}{\partial x_{1}}\left(0, x_{2}\right)= \begin{cases}1, & x_{2} \neq 0 \\ 0, & x_{2}=0\end{cases}
$$

Remark 6 An easy example on $\mathbb{R}$ shows that the Darboux property fails for vector-valued functions; the counterexample is given by $f$ which is defined as the antiderivative of

$$
f^{\prime}(x)= \begin{cases}\left(\cos \frac{1}{x}, \sin \frac{1}{x}\right), & x \neq 0 \\ 0, & x=0 .\end{cases}
$$

cf. [D], Problem 8.5.4. 


\section{References}

[B] A. M. Bruckner, Differentiation of Integrals, Supplement to Amer. Math. Monthly 78,9, Part II (1971).

[D] J. Dieudonné, Foundations of Modern Analysis, Academic Press, New York and London 1969.

[M] L. Mišik, Der Mittelwertsatz für additive Intervallfunktionen, Fund. Math. 45 (1957), 64-70.

[N] C. J. Neugebauer, Darboux property for functions of several variables, Trans. Amer. Math. Soc. 107 (1963), 30-37.

[Ph] R. R. Phelps, Convex Functions, Monotone Operators and Differentiability, Lecture Notes in Math. 1364, Springer-Verlag, Berlin 1993.

[W] C. E. Weil, A topological lemma and applications to real functions, Pacific J. Math. 44,2 (1973), 757-765. 